https://doi.org/10.18778/2196-8403.2021.10

YANG YAQING

JAROSŁAW APTACY

\title{
Korpusgestützte Analyse der Verben ernennen und 任命 (renming): ein fremdsprachenunterricht- orientierter deutsch-chinesischer Vergleich
}

Die Korpuslinguistik kann dem Fremdsprachenunterricht dienen und die Lernenden beim Wortschatzerwerb unterstützen. Davon ausgehend werden in dem vorliegenden Beitrag Sätze mit ernennen aus dem DWDS-Korpus und die mit 任命 (renming) aus dem BCC-Korpus exzerpiert und vor allem in Bezug auf ihre Valenzmuster vergleichend analysiert. Obwohl die zwei Wörter jeweils in ihrem eigenen Sprachsystem dasselbe bedeuten, unterscheiden sie sich merklich auf der parole-Ebene. Diese Unterschiede können nach unserer Ansicht Ursache für eine negative Interferenz in der Zielsprache sein. Durch eine Analyse der Valenzrealisierung dieser Verben konnten direkte Schlussfolgerungen für eine didaktische Aufarbeitung des untersuchten Materials gezogen werden.

Schlüsselwörter: Valenzrealisierung, Diathese, Fremdsprachendidaktik, Kollokation, Deutsch, Chinesisch

A corpus-based analysis of the verbs ernennen and 任命 (renming, English: to appoint) - a German-Chinese comparison based on foreign language teaching

Corpus linguistics can aid foreign language teaching and support vocabulary acquisition. With this assertion as a point of departure, this study is based on extracting sentences containing ernennen from the DWDS corpus, and those containing 任命 (renming) from the BCC corpus, in order to conduct a comparative analysis, mainly in terms of valency patterns. Although the two words each mean the same thing in their own language system, they differ noticeably at the parole level. In the Authors' opinion, these differences can cause negative interference in the target language. The analysis of the valence realization of these verbs allows clear conclusions to be drawn for didactic uses of the examined material. 
Keywords: valency realization, diathesis, language teaching, collocation, German, Chinese

Korpusowa analiza czasowników ernennen i 任命 (renming, poln. mianować) - porównanie niemiecko-chińskie w świetle dydaktyki języka obcego

Językoznawstwo korpusowe może służyć nauce języków obcych i wspierać uczniów w przyswajaniu słownictwa. Wychodząc $\mathrm{z}$ tego założenia, w niniejszym artykule dokonujemy porównawczej analizy niemieckich zdań z ernennen z korpusu DWDS i zdań chińskich z 任命 (renming) z korpusu BCC, głównie pod kątem struktur walencyjnych. Mimo że obydwa czasowniki znaczą to samo we własnym systemie językowym, różnią się one znacząco na poziomie parole. Naszym zdaniem różnice te mogą być przyczyną negatywnej interferencji w języku docelowym. Dzięki analizie realizacji walencji tych czasowników wyciągnięto bezpośrednie wnioski dla dydaktycznego wykorzystania badanego materiału.

Słowa klucze: realizacja walencji, diateza, nauczanie języka, kolokacja, język niemiecki, język chiński

\section{Einleitung}

Man bezeichnet als Korpuslinguistik die Beschreibung von Äußerungen natürlicher Sprachen, ihrer Elemente und Strukturen, und die darauf aufbauende Theoriebildung auf der Grundlage von Analysen authentischer Texte, die in Korpora zusammengefasst sind. [...] Korpusbasierte Sprachbeschreibung kann verschiedenen Zwecken dienen, zum Beispiel dem Fremdsprachunterricht [Hervorhebung der Autoren], der Sprachdokumentation, der Lexikographie oder der maschinellen Sprachverarbeitung bzw. Computerlinguistik. (LEMNITZER / ZINSMEISTER 2015:14f.)

Auf der Grundlage von Datenbasen können Linguisten Sprachen oder Tendenzen in der Sprachentwicklung unter verschiedenen Gesichtspunkten beobachten oder schon vorhandene Beschreibungen überprüfen und eventuell modifizieren. Dank der Korpora besitzt man neue Methoden, die Spracheinheiten und deren Strukturen zu beschreiben und zu interpretieren. Auch im Fremdsprachenunterricht finden die Forschungsergebnisse der Korpuslinguistik breite Anwendung. SINCLAIR / RENOUF (1988) bemerkten, dass die Lernenden einer Fremdsprache durch das Glossar in der Regel nur die Basisform der Wörter erlernen können. Traditionell erwerben die Lernenden Vokabeln und Grammatik separat. Von den Lernenden wird erwartet, dass sie die Wörter mittels der erworbenen grammatischen Regeln flektieren. Entsprechend bestimmten Konzepten von Lehrwerken und Unterrichtsgestaltungen werden Lücken im Satz gelassen, die mit geeigneten Wörtern / Wortformen gefüllt werden können. Mit anderen Worten, wenn man die Basisform der Wörter und die grammatischen Regeln 
der Zielsprache erworben hat, sollte man in der Lage sein, die Wörter sowohl morphologisch als auch syntaktisch korrekt anzuwenden. Aber in Wirklichkeit sind solche grammatisch korrekt flektierten Wörter in der Kommunikation vielfach kaum verwendbar. Davon ausgehend schlugen SINCLAIR / RENOUF (1988:148, 151) zum ersten Mal vor, dass die Vermittlung der Vokabeln im Fremdsprachenunterricht auf der Kernbedeutung und auf Kollokationen ${ }^{1}$ mit hoher Frequenz ${ }^{2}$ basieren sollte. Danach haben Sprachwissenschaftler Versuche unternommen, Korpora mit dem Vokabellernen zu kombinieren und dabei beachtliche Leistungen erbracht. Sie haben entweder einen lexikalischen Lehrplan (engl. lexical syllabus) nach der Idee von SINCLAIR / RENOUF entworfen (WILLIS 1990; WILLIS / WILLIS 1990), oder das Konzept des datengesteuerten Lernens (engl. data-driven learning) vorgeschlagen (JONAS 1991). Im Falle der chinesischen Englischlerner wurden anhand des Chinese Learner English Corpus (kurz: CLEC) Probleme und Schwächen der Lerner beim Vokabellernen untersucht. (PU 2003, vgl. ZHEN / YANG 2016)

\section{Valenzpattern für Fremdsprachenunterricht}

In früheren Studien wurde bestätigt, dass man Korpora und Forschungsergebnisse der Korpuslinguistik im Fremdsprachenunterricht einsetzen kann, zum Beispiel bei dem Entwerfen von Lehrwerken oder der Unterrichtsgestaltung.

ZHEN / YANG $(2015,2016)$ integrierten die Untersuchungsmethodik von pattern grammar, die im Rahmen der Korpuslinguistik die grammatischen Strukturen relativ systematisch beschreibt, und der Valenzgrammatik, die viel Wert auf die syntaktische Funktion legt, um Zusammenhänge zwischen Lexik und Grammatik zu ermitteln. Mit dieser integrierten Untersuchungsmethode haben sie das Wort consider als Beispiel genommen und seine Valenzpatterns mit ihrer Frequenz im CLEC analysiert und dann mit den Daten aus dem Korpus der Englisch-Muttersprachler (British National Corpus $=$ BNC) verglichen. Die

1 Unter Kollokation werden hier in Anlehnung an STOPYRA (2019:78) „,binär aufgebaute Phrasen verstanden, bei denen die Kookkurrenz (Sequenz) der Komponenten üblich ist und die einen bestimmten Grad der Festigkeit aufweisen. Die prototypischen Kollokationen weisen eine Prädikat-Argument-Struktur auf und bestehen aus zwei Autosemantika.“

2 Nach dem Birmingham Corpus, auf das sich die Autoren beziehen, weisen diejenigen (ggf. flektierten) Wortformen eine hohe Frequenz auf, die sich unter den ca. 800 ersten Wortformen auf der Rankingliste befinden. 
Einsicht, dass einige Patterns in den Äußerungen der Muttersprachler häufig auftreten, dagegen sehr selten oder kaum in denen der Lernenden, oder umgekehrt, kann der Lehrkraft dabei helfen, neben dem Glossar mit Basisformen entsprechende Valenzpatterns von großer Häufigkeit zu vermitteln.

Bei der Analyse des Lernerkorpus haben ZHEN / YANG die passivischen Sätze, in denen das Verb consider vorkommt, aus folgenden Gründen ausgefiltert: (1) Die meisten bisherigen Valenzstudien basieren auf unmarkierten aktivischen Sätzen, die passivischen gelten als abgeleitet; (2) nach ihren Beobachtungen ändert sich die Valenz des Verbs consider nicht, wenn es von passivischen in aktivische Sätze umgewandelt wird. Aber wie sie selbst einräumten, gehört zur Kollokationsstruktur eines Verbs nicht nur die Zahl und Art der von ihm beherrschten Ergänzungen und Angaben, sondern auch, ob das Verb im Passivsatz vorkommt. LIU / DU (2017) führten anhand der Methodik, die ZHEN / YANG vorgeschlagen hatten, Genus verbi ein, um die grammatikalische Struktur der Lexeme, v.a. der Verben, umfassender zu analysieren. Sie nahmen das Verb appoint als Beispiel und beschrieben nach den Korpusdaten sein Valenzpattern in Aktiv- und Passivsätzen sowie anderen Satzformen. Die Ergebnisse dieser Studie haben Implikationen für die Vermittlung des englischen Wortschatzes auf Universitätsniveau, indem die Kollokations- und semantischen Sequenzanalysewerkzeuge der Korpuslinguistik zur Analyse von Subjekt, Objekt und anderen dominanten Valenz-Komponenten des Wortes verwendet werden.

Dieses Analyseparadigma kann nicht nur im Englischunterricht eingesetzt werden, sondern auch im Unterricht anderer Fremdsprachen. Wenn wir beispielsweise den Deutschunterricht beobachten, sind - wie oben erwähnt - die Vermittlung von Lexik und Grammatik zwei relativ getrennte Lehrhandlungen. Aber aus früheren Studien ist leicht zu erkennen, dass das Valenzpattern und die Bedeutung des Verbs in einer wechselseitigen Auswahlbeziehung stehen. Wenn sich das Valenzpattern eines Verbs ändert, ändern sich auch die Kollokationen und die ausgedrückte Bedeutung ändert sich dementsprechend. Mit anderen Worten, die Wortbedeutung ist mit dem Valenzpattern und den Kollokationen eng verknüpft (ZHEN / YANG 2016). In der vorliegenden Studie wird das Verb ernennen als Beispiel genommen. Anhand des deutschen Korpus DWDS werden verschiedene Valenzpatterns beschrieben und ihre jeweilige Frequenz analysiert. Die Ergebnisse werden dann mit den Ergebnissen des Valenzpatterns des entsprechenden chinesischen Wortes 任命 (renming, ernennen) in chinesischen Korpusdaten aus $\mathrm{BCC}^{3}$ verglichen, um Präferenzen der Sprach-

Ein an der Beijing Language and Culture University entwickeltes Korpus. 
verwendung in beiden Sprachen festzustellen. Da wir davon ausgehen, dass die Muttersprache negative Interferenz auf das Deutschlernen ausüben kann, könnten die Ergebnisse der vorliegenden Untersuchung nach geeigneter didaktischer Aufarbeitung zur effektiveren Wortschatzvermittlung im Deutschunterricht beitragen und u.a. dabei helfen, die Fehlanwendung der Kollokationen zu vermeiden und präzisere sprachliche Formulierungen zu fördern.

\section{Forschungsdesign}

Die Untersuchung dient dazu, die folgenden Fragen zu beantworten:

- Welche Valenzpatterns des deutschen Verbs ernennen bzw. des chinesischen Verbs renming kommen im jeweiligen Sprachsystem vor?

- Wie unterscheiden sich die Anwendungen von ernennen und renming im Hinblick auf Valenzpatterns?

- Wie kann man der negativen Interferenz der Muttersprache Chinesisch bei dem Entwurf eines Lehrwerks und der Gestaltung des Deutschunterrichts vorbeugen?

Um diese Fragen zu beantworten, werden zuerst zwei parallele Korpora des Deutschen und Chinesischen jeweils mit ernennen und renming aufgebaut. Sie werden dann manuell bearbeitet: d.h. sie werden annotiert und es wird eine Konkordanzerstellung durchgeführt.

\subsection{Download und Annotation}

Das DWDS-Kernkorpus des 21. Jahrhunderts ist ein zeitlich und nach Textsorten (Belletristik, Gebrauchsliteratur, Wissenschaft, Journalistische Prosa) differenziertes, momentan aber noch nicht ausgewogenes Korpus. Es enthält belletristische und wissenschaftliche Texte aus den Jahren 2000 bis 2006 sowie Zeitungstexte. (Startseite DWDS)

Alle deutschen Belege in diesem Aufsatz entstammen dem DWDS-Kernkorpus 21. Um den Gebrauch des Verbs ernennen zu untersuchen, wurden alle 153 Sätze, die das Verb enthalten, heruntergeladen, und dann manuell annotiert. Bei der Definition der Tagsets haben wir uns vor allem auf grammatische Kategorien gestützt. Mithilfe der Tags_Akt und_Pass lassen sich Aktiv- und Passivsätze unterscheiden. $\mathrm{Ob}$ das Verb ein zweiwertiges Verb mit einer Subjektivund Akkusativergänzung oder ein dreiwertiges Verb mit einer zusätzlichen durch $z u$ eingeleiteten Präpositionalergänzung ist, unterscheidet der Tag_Akk und_Präp. Tags in Hinsicht auf Tempus, wie _Präs,_Perf, _Prät, u.a., und in 
Hinsicht auf Modus, wie _Konj, und andere, wie _MV und _Inf, stehen zur tempus- sowie modusbezogenen Untersuchung dieses Verbs zur Verfügung.

Bei der Annotation wurden fünf Sätze ausgesiebt, weil ihnen aufgrund des Kontexts das Hilfsverb fehlte. Obwohl man problemlos erkennen kann, dass diese Sätze passivisch sind, bleiben Tempus und Modus, die aber zur Analyse der Daten benötigt werden, verdeckt. Deshalb war es sinnvoller, diese Sätze ohne Hilfsverben bei der Datenanalyse auszuschließen. Folglich wurden in dieser Arbeit insgesamt 148 von 153 Treffern für den Suchbegriff ernennen bearbeitet.

Die chinesischen Sätze mit renming stammen vom Korpus BCC.

Das BCC ist ein Online-Korpus mit zig Milliarden Wörtern aus dem Chinesischen bzw. anderen Sprachen. Es dient zur sprachontologischen Forschung und der Sprachanwendungsforschung. Sein Ziel ist es, ein einfach zu bedienendes OnlineRetrieval-System für die Recherche in Bezug auf Sprachontologie bereitzustellen und eine Basisplattform für Big-Data-Sprachanwendungen aufzubauen. (vgl. XUN ET. AL. 2016) ${ }^{4}$

Um die Analyse symmetrisch zu gestalten, werden aus dem Korpus BCC 148 Sätze, genauso viele wie aus dem deutschen Korpus, zufällig ausgewählt. Sie werden in gleicher Weise wie oben erwähnt annotiert, vor allem im Hinblick auf Genus verbi und Valenzpatterns. Das Tempus wird nur annotiert, wenn es nicht Präsens ist. Die daraus stammenden Angaben über Formen und Frequenzen bilden die Grundlage für die weitere Analyse.

\subsection{Konkordanzerstellung}

Mit dem Konkordanzprogramm AntConc wurden die Konkordanzen erstellt. Mit diesem Programm ist es möglich, zu zählen, wie oft ernennen als Vollverb, und wie oft es als Attribut in Form eines Partizip II verwendet wird, und zu betrachten, ob die gegebene Struktur unter bestimmten Bedingungen öfter gebraucht wird.

Wegen der technischen Mängel ist es nicht möglich, die chinesischen Daten mit AntConc auszuwerten. Daher wird die chinesische Konkordanz hauptsächlich mit Word bearbeitet und manuell ausgewertet.

$4 \quad$ Übersetzt von den Autoren. 


\section{Datenanalyse des deutschen Verbs ernennen}

\subsection{Genus verbi}

Ernennen kann nicht nur als Vollverb im Satz verwendet werden, sondern auch als Attribut in Form eines Partizip II, dekliniert wie Adjektive. Die Frequenzverteilung im Korpus ist in Tabelle $1 \mathrm{zu}$ sehen.

Tabelle 1. Frequenzverteilung der Formen von ernennen

\begin{tabular}{|c|c|c|c|}
\hline \multicolumn{2}{|c|}{ Vollverb } & \multicolumn{2}{c|}{ Partizip II } \\
\hline Aktiv & Passiv & Attribut & Sonderfall \\
\hline 64 & 69 & 14 & 1 \\
\hline $43 \%$ & $47 \%$ & $9 \%$ & $1 \%$ \\
\hline
\end{tabular}

Die Tabelle zeigt, dass man ernennen im Aktiv fast so oft wie im Passiv verwendet. Es kommt auf den textuellen Kontext an, der entscheidet, ob Agens oder Patiens als bekannte Information an die Stelle des Topik an den Satzanfang gesetzt werden soll.

Der Anteil der Partizip II-Formen beträgt etwa $10 \%$. Die große Mehrheit davon fungiert als Attribut vor dem Nomen. Vor zehn von 14 attributiven Partizipien steht selbst (Beispiel 1). Bei den übrigen wird durch vom Staat (Beispiel 2) und zum Ehrenbürger (Beispiel 3) deutlich, wer ernennt, und zu was ernannt wird.

1. der selbst ernannte Hobbyarchäologe

2. das neue, vom Staat ernannte Management von Studio B

3. der 1994 zum Ehrenbürger ernannte Sportler

Es ist anzumerken, dass der Handelnde und das Resultat der Ernennungshandlung bei der Anwendung des Verbs eine große Rolle spielen, weil man schon beim ersten Hören des Wortes ernennen Informationen darüber erwartet. Welche Informationen vermittelt werden, ist mit dem Valenzpattern eng verbunden, das im nächsten Teilkapitel beschrieben wird. 


\subsection{Valenz}

Im Online-Wörterbuch Duden steht, dass das Wort ernennen zwei Bedeutungen hat ${ }^{5}$, nämlich a. für ein Amt, einen Posten bestimmen, zum Beispiel: jemanden zum Vorsitzenden ernennen; b. den Inhaber bzw. die Inhaberin eines Amtes bestimmen, zum Beispiel: einen Nachfolger ernennen. Demgemäß hat das Verb ernennen zwei Gebrauchsformen. Wie auch durch das Korpus bestätigt wird, ist ernennen zweiwertig (Beispiel 4) oder dreiwertig (Beispiel 5).

4. Außerdem ernannte der Präsident neue Minister für Handel sowie für Investitionskontrolle [...].

5. Staatspräsident KIM Dae Jung ernannte am 11. Januar den Manager und Vorsitzenden der Regierungspartei ULD, PARK Tae Joon, zum neuen Ministerpräsidenten Südkoreas [...].

Im zweiwertigen Aktivsatz ist außer dem Subjekt (Theta-Rolle: Agens) eine Akkusativergänzung (Theta-Rolle: Patiens) notwendig. Im Beispiel 4 ist sie neue Minister für Handel sowie für Investitionskontrolle. Die Korpusdaten ergeben, dass das Patiens entweder eine Stelle ist, die von einer Person besetzt wird (Beispiel 6), oder ein Amt darstellt, das hauptsächlich eine politische oder exekutive Funktion hat (Beispiel 7).

6. Die honduranische Regierung ernannte daraufhin 1998 einen neuen Tourismusminister [...].

7. Die Regierungspartei wies die EU-Kritik zurück; sie hatte noch vor Bekanntgabe des Wahlergebnisses betont, dass MUGABE, der bis 2002 Präsident bleibt, auch die nächste Regierung ernennen werde.

Beim dreiwertigen Gebrauch kommt neben dem Agens und Patiens noch eine Präpositionalergänzung mit $z u$ hinzu (Beispiel 8):

8. Zum neuen Wirtschafts- und Finanzminister ernannte er JIN Nyum, den bisherigen Chef des Haushalts- und Planungsressorts.

In dieser Struktur sind die Informationen noch weiter vervollständigt und bestehen jetzt aus:

5 Bedauerlicherweise konnte in den üblichen Valenzlexika der deutschen Verben kein Eintrag für ernennen gefunden werden. Dies gilt auch für das fremdsprachdidaktisch orientierte und sehr inhaltsreiche Valbu-Lexikon (vgl. SCHUMACHER ET AL. 2004). 
1) einer Person / einer Organisation / einer Institution, die die Macht einer (anderen) Person ${ }^{6}$ verleiht;

2) einer Person, die die Macht erhält;

3) einer Stelle oder Position, die diese Person nach der Ernennung innehaben soll.

Daher kann man die folgende Tabelle erstellen, die den Zusammenhang der Valenzpatterns und der betreffenden Informationen zeigt:

Tabelle 2. Valenzpatterns und betreffende Informationen von ernennen

\begin{tabular}{|l|l|l|l|}
\hline Valenzpattern & \multicolumn{1}{|c|}{ Agens } & \multicolumn{1}{|c|}{ Patiens } & \multicolumn{1}{c|}{ PP } \\
\hline Zweiwertig & $\begin{array}{l}\text { - Organisation / Institution } \\
\text { - hochrangige Persönlichkeit }\end{array}$ & $\begin{array}{l}\text { - Stelle } \\
\text { - Amt }\end{array}$ & - \\
\hline Dreiwertig & $\begin{array}{l}\text { - Organisation / Institution } \\
\text { - hochrangige Persönlichkeit }\end{array}$ & - Person & - Stelle \\
\hline
\end{tabular}

Die Tabelle 2 stellt den Zusammenhang zwischen vermittelten Informationen und Valenzpatterns dar. Wenn man die semantischen Rollen von den beiden Valenzpatterns vergleicht, bemerkt man, dass bei Dreiwertigkeit die Bezeichnung derjenigen Person, die die Macht annimmt, hinzugefügt wird. Aber diese Bezeichnung raubt dem Satz die Information über die Stelle, die bei Zweiwertigkeit als Patiens auftritt. Diese muss dann in die Präpositionalphrase mit $z u$ verschoben werden.

Gemäß dem Korpus besteht die Tendenz, durch Zusatz der Präpositionalergänzung vollständige Informationen anzubieten. Die Daten im Kreisdiagramm in Abbildung 1 zeigen, dass die Häufigkeit mit $z u$ fast doppelt so hoch ist.

6 Es ist nicht immer der Fall, dass die Person, die die Macht bekommt, eine andere ist, da es manchmal durch ein reflexives Pronomen zum Ausdruck kommt, dass diese Person auch die gleiche ist wie die, die die Macht verleiht. Ein Beispiel: [...], da Ich Mich [...] zum alleinigen Inhaber der obengenannten Inseln ernenne. Auch eine reflexive dreiwertige Struktur ist möglich: [...]; unser Jonathan Lambert hat sich bloß zur falschen Zeit zum König ernannt. 


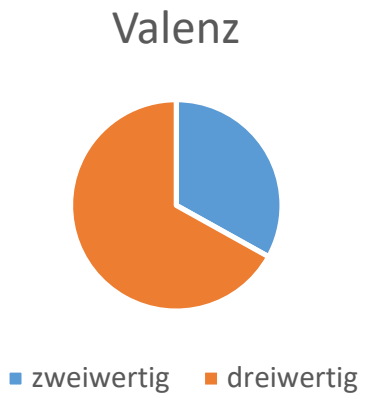

Abbildung 1. Valenzverteilung zweiwertig : dreiwertig.

Wie im letzten Unterabschnitt erwähnt wurde, ist die Frage: „Zu was wird jemand ernannt?" wichtig, da man beim Einsatz von ernennen diese Information erwartet. Die vorliegenden Daten können das bestätigen.

Bei näherer Beobachtung stellt sich heraus, dass sich die Frequenz der Realisierung von Zweiwertigkeit und Dreiwertigkeit im Aktiv und Passiv unterscheidet.

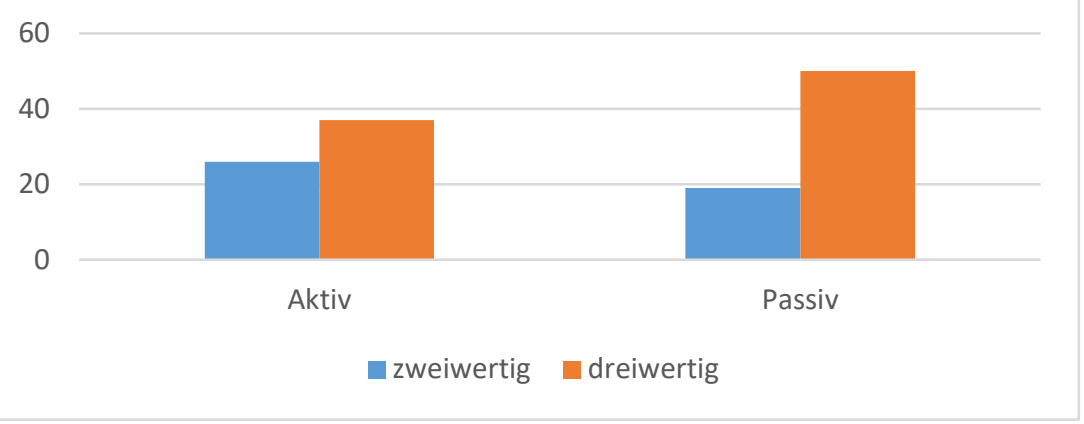

Abbildung 2. Valenzverteilung im Aktiv und Passiv.

Im Passiv scheint die Information über die Stelle der Machtübertragung noch wichtiger. Normalerweise fungiert das Patiens im Passivsatz als eine bekannte Information. Die Bildung eines Passivsatzes dient in der Regel funktional als Kohäsionsmittel, als Zeichen dafür, dass das Patiens Topik dieses Satzes ist und demnach Ausgangspunkt der Aussage. Über die Person, die ernannt wird, 
werden neue Informationen angegeben. Was sie wird, wird durch die präpositionale Ergänzung mit $z u$ als Fokus eingeführt.

\subsection{Tempus und Modus}

Nach der Auszählung der Tempusformen sowie Strukturen mit Modalverben erhält man folgendes Bild:

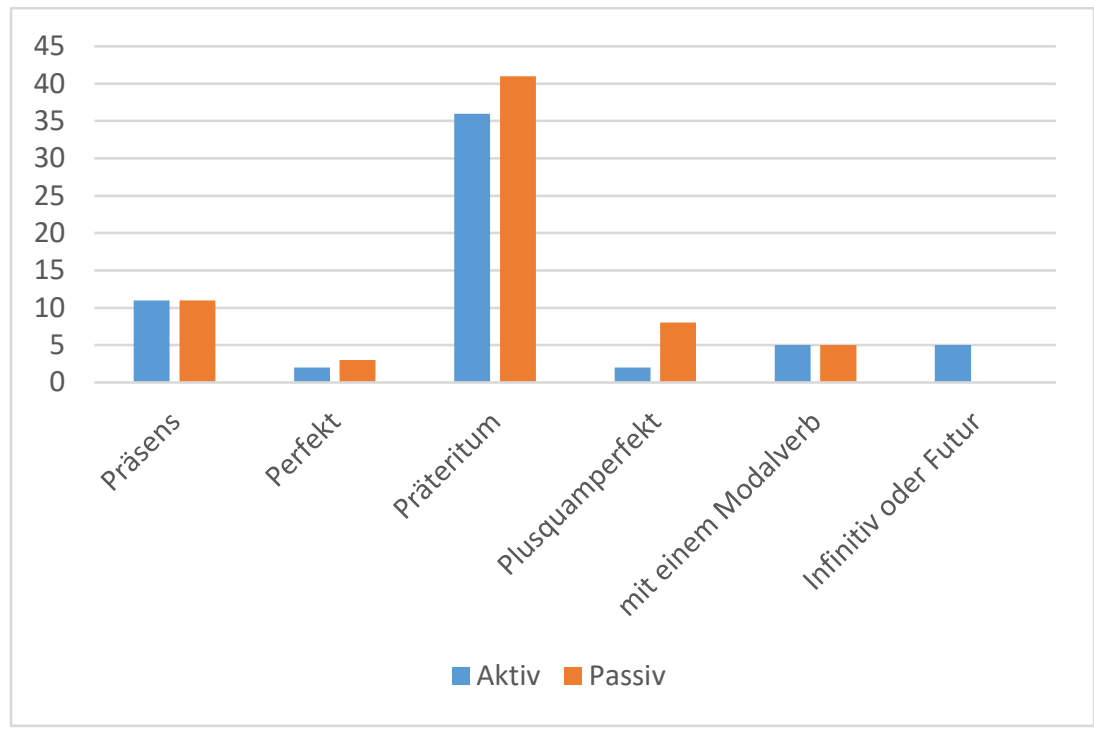

Abbildung 3. Verteilung von Tempus und modalverbhaltigen Strukturen.

Das Säulendiagramm in Abbildung 3 veranschaulicht Folgendes:

1) Sowohl im Aktiv als auch im Passiv wird ernennen mit großem Abstand öfter im Präteritum als in anderen Tempora verwendet.

2) Die Perfektform kommt selten vor.

3) Ernennen im Infinitiv oder Futur verwendet man sehr selten im Aktiv, und in diesem Korpus gar nicht im Passiv.

Der Grund für den häufigsten Einsatz von Präteritum könnte darin liegen, dass man sich beim Gebrauch von ernennen oft auf ein Ereignis in der Vergangenheit bezieht, und dass die Textsorten, in denen dies auftritt, meistens berichtenden Charakter haben, für den wiederrum das Präteritum kennzeichnend ist. Das führt dazu, dass man bei der Verwendung des Verbs lieber Präteritum als 
Perfekt einsetzt. Aber Plusquamperfekt kommt auch manchmal vor, weil es in bestimmten Kontexten grammatisch bedingt ist; dadurch stellt man eine Handlung dar, die vor einer anderen Handlung in der Vergangenheit stattgefunden hat. Punkt 3) können wir aufgeteilt analysieren, wobei es zuerst um den Infinitiv geht. In den Beispielen folgt ernennen Verben oder verbalen Wendungen wie vorschlagen, das Recht geben und vorsehen (Beispiel 9), oder als Rechtsattribut nach einem Nomen wie Entscheidung (Beispiel 10). Bei solchen Ausdrücken soll meistens das Patiens des Verbs, nämlich wer die Macht bekommt, im Fokus stehen. Der Infinitiv im Aktiv ermöglicht diese Funktion.

9. [...] da die Verfassung Mugabe das Recht gebe, eine Regierung ohne Berücksichtigung der Zusammensetzung des Parlaments zu ernennen.

10. Die Entscheidung Bushs, Cheney selbst zum Vizepräsidentschaftskandidaten zu ernennen [...].

Als Nächstes wird ernennen im Futur besprochen. Im Deutschen kann man sich oft im Präsens mit einer temporalen Angabe über ein Ereignis in Zukunft äußern. Mit Futur kann man den Wunsch und den Willen der Person, die die Macht verleiht, in den Vordergrund rücken. In diesem Sinne ist es notwendig, dass das Agens als Topik im Aktiv auftritt.

Zu erwähnen sind auch Sätze im Konjunktiv (Konjunktiv in Beispiel 11), die nur einen geringen Teil ausmachen (in dem vorliegenden Korpus nur sechs Sätze), ansonsten wird die Modalität (vier von sechs) durch Modalverben (Beispiel 12) realisiert.

11. [...] da [...] der junge König klerikalem Druck nachgegeben hatte und ihn, den Großen d'Albuquerque, nicht den Bonvivant da Cunha, auf geheimen Befehl zum Vizekönig in Indien ernennen würde.

12. Sollten die Vereinten Nationen Dr. Naumann zum globalisierten Weltkulturminister ernennen [...], könnte der Europarat Dr. Christoph Stölzl zum Europakulturminister mit Schwerpunkt nationale Sitten und Gebräuche befördern $[\ldots]$.

Tabelle 3. Frequenzverteilung von Konjunktiv I und Konjunktiv II

\begin{tabular}{|c|c|c|c|c|c|c|c|}
\hline \multicolumn{3}{|c|}{ Konjunktiv I (insgesamt 2) } & \multicolumn{3}{c|}{ Konjunktiv II (insgesamt 4) } \\
\hline \multicolumn{2}{|c|}{ Genus verbi } & \multicolumn{2}{|c|}{ Valenz } & \multicolumn{2}{c|}{ Genus verbi } & \multicolumn{2}{c|}{ Valenz } \\
\hline Aktiv & Passiv & zweiwertig & dreiwertig & Aktiv & Passiv & zweiwertig & dreiwertig \\
\hline 0 & 2 & 1 & 1 & 3 & 1 & 2 & 2 \\
\hline
\end{tabular}


Es gibt insgesamt 2 Sätze im Konjunktiv I und 4 im Konjunktiv II. Wie die Tabelle zeigt, kann ernennen im Konjunktiv II im Aktiv oder Passiv gebraucht werden, auch beide Valenzpatterns von Zwei- und Dreiwertigkeit sind zugänglich. Aber die Anzahl der Belege ist zu gering, um durch die Häufigkeit jener Form Tendenzen im Gebrauch zu beschreiben. Außerdem ist es bemerkenswert, dass beide Beispiele im Konjunktiv I (Beispiel 13, 14) passivisch sind.

13. BASSAJEW sei zum Kommandeur der Rebelleneinheiten und der jordanische Söldner KHATTAB zu seinem Stellvertreter ernannt worden.

14. Der neue Premier Jugoslawiens müsse entsprechend der Verfassung ernannt werden, die fordere [...]

Es könnte sein, dass man mehr auf die Person, der die Macht gegeben wird, fokussiert, wenn man das Ereignis wiedergibt. Aber auch wegen der geringen Beleganzahl ist eine solche Hypothese anhand einer größeren Datenmenge zu überprüfen.

\section{Analyse des chinesischen Verbs renming}

\subsection{Genus verbi}

Da die chinesische Sprache zum isolierenden Sprachtyp gehört, hat Chinesisch im Gegensatz zu der flektierenden deutschen Sprache keine Flexion. Die Grenzen zwischen Wortarten sind nicht deutlich. Die gegenwärtigen chinesischen Linguisten neigen dazu, Wörter eher nach ihrer Funktion im Satz als nach Wortarten zu klassifizieren. Wenn man das chinesische Korpus näher betrachtet, ist zu bemerken, dass renming nicht nur als Verb im Satz, sondern auch als Bestandteil von Nominalphrasen (NP) fungieren kann. Die Distribution sieht wie in Tabelle 4 aus:

Tabelle 4. Frequenzverteilung von renming

\begin{tabular}{|c|c|c|c|}
\hline \multicolumn{2}{|c|}{ Verb } & \multicolumn{2}{c|}{ Bestandteil von einer NP } \\
\hline Aktiv & Passiv & Kernwort einer NP & Attribut einer NP \\
\hline 65 & 32 & 20 & 31 \\
\hline $44 \%$ & $22 \%$ & $13 \%$ & $21 \%$ \\
\hline
\end{tabular}


Es ist nicht überraschend, dass die Funktion als Bestandteil einer NP häufiger vorliegt als im Deutschen, weil die Wortform renming im Chinesischen als Verb, Adjektiv und Nomen gebraucht werden kann (siehe Beispiel 15 (Verb), 16 (Bestimmungswort eines substantivischen Kompositums), 17 (Adjektiv), 18 (Nomen)).
15. Aerjiliya
renming
xin
zongli.
Algerien
ernennen
neu Ministerpräsident.

$\rightarrow \quad$ Algerien ernannte einen neuen Ministerpräsidenten.

16. renmingzhi -> Ernennungssystem

17. you zhengfu renming de guanyuan $->$ von der Regierung ernannter Beamter 18. Dan zhongguo zuxie de zhengshi renming haiyao aber CFA de offizielle Ernennung noch deng jitian cai hui chutai. in einigen Tagen erst vorliegen

$\rightarrow$ Aber die offizielle Ernennung durch die CFA wird erst in einigen Tagen vorliegen.

Trotzdem zeigen die Daten, dass renming meist als Verb auftritt, und zwar doppelt so häufig in Aktivsätzen wie in Passivsätzen.

Im chinesischen Korpus gibt es einige wenige Satzstrukturen mit spezifisch chinesischen grammatischen Erscheinungen.

19. Ta ba jinke abandandu renming wei canmou. er ba Genco Abbandando ernennen $\mathrm{zu}$ Stabsoffizier.

$\rightarrow$ Er ernannte Genco Abbandando zum Stabsoffizier.

20. ta renming jinke abandandu wei canmou. er ernennen Genco Abbandando $\mathrm{zu}$ Stabsoffizier.

$\rightarrow$ Er ernannte Genco Abbandando zum Stabsoffizier.

21. Qi juzhang you zongli Der Direktor YOU Ministerpräsident zhijie renming. unmittelbar ernennen.

$\rightarrow$ Der Direktor wird unmittelbar von dem Ministerpräsidenten ernannt. 
Beispiel 19 ist ein $b a$-Satz, der in einen unmarkierten SVO-Satz ${ }^{7}$ mit renming als Vollverb umformuliert werden kann, wie im Satz 20. Beide Sätze vermitteln die gleiche Information, unterscheiden sich aber durch ihre kommunikative Funktion. Mit einem $b a$-Satz betont der Sprecher das Handlungsergebnis oder die Handlungsweise. Beispiel 21 ist ein Passivsatz. Normalerweise werden Passivsätze mit der Präpositionalphrase ${ }^{8}$ mit 被 (-bei) gebildet. Im Korpus treten auch Passivsätze wie Beispiel 21 auf, die mit Präposition 经 (-jing, durch), oder 由 (-you, durch) gebildet werden. Davon sind die meisten Sätze zweiwertig, ganz im Gegensatz zu bei-Sätzen, in denen renming eher dreiwertig ist.

\subsection{Valenz}

Wie im Deutschen hat das chinesische Verb renming auch die zweiwertige oder dreiwertige Valenz. Für Zweiwertigkeit können wir Satz 15 oben als Beispiel nehmen, in dem Subjekt und Objekt vorkommen. Bei Dreiwertigkeit kommt noch eine Präpositionalphrase mit 为 (-wei, zu) hinzu (Beispiel 22). Auffallend ist die Pivotalkonstruktion mit einer eingebetteten Verbphrase, die die Informationen über die Position der ernannten Person bzw. Institution o.ä. angeben soll. (Beispiel 23)

22. gongsun shu renming wang yuan wei jiangjun ...

Gongsun Shu ernennen Wang Yuan $\mathrm{zu}$ General ...

$\rightarrow$ Gongsun Shu ernannte Wang Yuan zum General ...

23. bilishi guowang aibote ershi jintian renming shehui dang

$\begin{array}{llcc}\text { Belgischer } & \text { König } & \text { Albert II heute ernennen } & \text { Parti Socialiste } \\ \text { dihebo } & \text { lingdao } & \text { xin zhengfu. } & \end{array}$
di Rupo leiten die neue Regierung.

$\rightarrow$ Der Belgische König Albert II ernannte heute di Rupo von der Sozialistischen Partei zum Leiter der neuen Regierung.

Eine Pivotalkonstruktion hat zwei Prädikate, von denen das erste immer ein verbales Prädikat ist. Das Objekt des ersten Prädikats ist das Subjekt des zweiten. Es fungiert als „Achse“ (pivot im Französischen), die zwei Verbphrasen ver-

7 Im Gegensatz zur markierten SOV-ba-Diathese.

8 Die Wortart von 被(-bei) in bei-Sätzen ist ein umstrittenes Thema. Ist bei eine Partikel, ein Hilfsverb oder eine Präposition? Darüber streiten sich Linguisten schon seit langem. Hier vertreten wir die Ansicht, dass bei eine Präposition ist, die ein Substantiv begleitet und dabei eine passivische Bedeutung anzeigt. 
bindet. Im Beispiel 23 ist renming das verbale Prädikat, die ernannte Person di Rupo ist die Achse des Satzes, die sowohl Objekt des ersten Prädikats renming, als auch Subjekt des zweiten Prädikats lingdao ist. Renming in Pivotalkonstruktionen macht ungefähr $9 \%$ von allen Valenzpatterns aus, was im Deutschen wegen einer anderen Subkategorisierung von ernennen nicht gegeben ist.

Die Verteilung der drei Valenzpatterns sieht wie in Abbildung 4 aus.

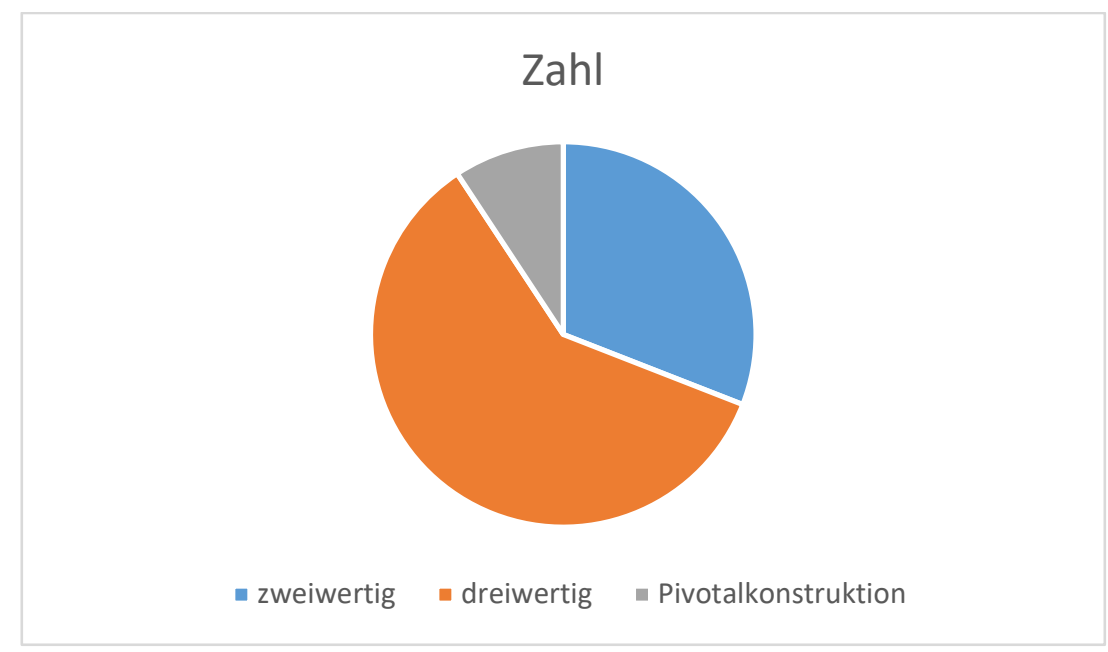

Abbildung 4. Valenzverteilung zweiwertig: dreiwertig: Pivotalkonstruktion.

Dreiwertige Konstruktionen machen 60 \% Prozent aus. Das bestätigt wieder, dass man mit renming eher vollständige Informationen vermitteln will. Wenn man eine semantische Analyse der drei Valenzpatterns durchführt, kommt man zu den folgenden Ergebnissen: 
Tabelle 5. Semantische Analyse der Valenzpatterns von renming

\begin{tabular}{|c|l|l|l|l|l|}
\hline Valenzpattern & \multicolumn{1}{|c|}{ Agens } & Patiens & PP & VP & $\begin{array}{c}\text { Aktiv: } \\
\text { Passiv }\end{array}$ \\
\hline Zweiwertig & $\begin{array}{l}\text { Organisation/Institution, } \\
\text { hochrangige } \\
\text { Persönlichkeit, Staats- } \\
\text { oder Ortsname }\end{array}$ & Stelle & - & - & $2: 1$ \\
\hline Dreiwertig & $\begin{array}{l}\text { Organisation/Institution, } \\
\text { hochrangige } \\
\text { Persönlichkeit }\end{array}$ & Person & Stelle & - & $3: 2$ \\
\hline $\begin{array}{c}\text { Pivotal- } \\
\text { konstruktion }\end{array}$ & $\begin{array}{l}\text { Organisation/Institution, } \\
\text { hochrangige } \\
\text { Persönlichkeit }\end{array}$ & Person & - & $\begin{array}{l}\text { - Stelle } \\
\text {-Institution } \\
\text { /Gruppe }\end{array}$ & $\begin{array}{l}\text { alle } \\
\text { aktiv }\end{array}$ \\
\hline
\end{tabular}

a) Bei Zweiwertigkeit ist das Agens eine Organisation/Institution, ein*e politischer Leiter*in, ein Staats- oder Ortsname. Das Patiens ist hauptsächlich die Stelle, die die ernannte Person antreten sollte. Ausnahmen sind nur zwei Sätze in unserem Korpus, einmal ist das Patiens eine bestimmte Person, einmal eine Institution. Es gibt doppelt so viele Aktivsätze wie Passivsätze.

b) Bei Dreiwertigkeit ist das Agens die Organisation/Institution oder der*die politische Leiter*in. Das Patiens ist eine bestimmte Person, aus der Präpositionalphase kann man die Stelle erfahren. In bestimmten Textsorten wie in einer Regierungsankündigung ist oft das Agens weggelassen, da es ohne Zweifel die Regierung ist, und weil es das kommunikative Ziel der Ankündigung ist, nur die ernannte Person und die Stelle anzugeben. Aus sprachökonomischen Gründen ist die Information über das Agens redundant. In Passivsätzen neigt man auch dazu, das Agens nicht ausdrücklich anzugeben. Solche Sätze machen 73 \% (16 von 22) aller dreiwertigen Passivsätze aus.

c) In der Pivotalkonstruktion sind alle Sätze mit renming aktiv. Genauso wie bei Dreiwertigkeit ist das Agens eine Organisation/Institution oder ein*e politische*r Leiter*in, und das Patiens ist eine bestimmte Person. Verben, die mit renming Pivotalkonstruktionen bilden, kann man in zwei Typen klassifizieren. a) Verben wie 担任 (-danren, antreten), 乔升为(-qiaoshengwei, befördern), 代 行...... 职务 (-daixing ... zhiwu, die Stelle als ... nehmen) mit der Stelle; b) Verben wie 领导 (-lingdao, leiten), 指挥(-zhihui, kommandieren) mit einer Institution oder Gruppe, für die die ernannte Person zuständig sein sollte. 


\subsection{Tempus}

Da Chinesisch keine Flexion hat, wird die zeitliche Charakteristik im Chinesischen nur mit Adverbien oder Partikeln zum Ausdruck gebracht. Darüber hinaus kann man die Zeit mit einer Zeitangabe wie gestern oder am 22. März o.ä. anführen, was in unserem Korpus bestätigt wird. Nur in sehr seltenen Fällen (4 \%) haben Sätze ein Adverb oder eine Partikel als Zeichen des Präteritums vor oder nach dem Verb (了, le in Beispiel 24).

$\begin{array}{lllll}\text { 24. Songxixian } & \text { xuanba } & \text { renming } & \text { le } & \text { xianyiyuan } \\ \text { Landkreis Songxi } & \text { auswählen } & \text { ernennen } & \text { le } & \text { Kreiskrankenhaus } \\ \text { yewu fuyuanzhang } & & & & \end{array}$

Stellvertretender Betriebsleiter

$\rightarrow$ Der Landkreis Songxi wählte aus und ernannte den Stellvertretenden Betriebsleiter des Kreiskrankenhauses.

\section{Fremdsprachendidaktische Implikationen}

Wenn man die Belege mit den Verben ernennen und renming vergleicht, ist leicht zu erkennen, dass sich der Gebrauch dieser zwei Verben unterscheidet.

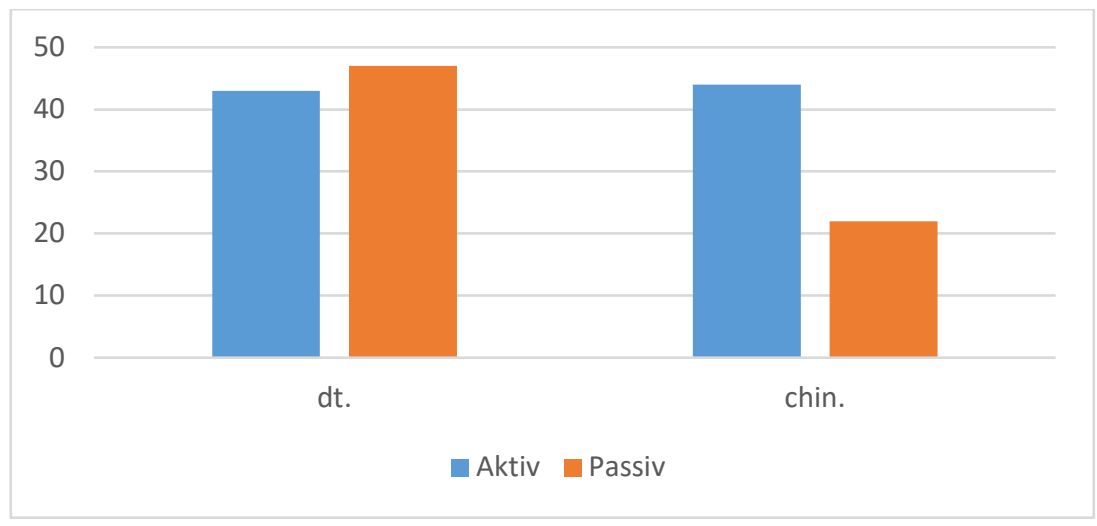

Abbildung 5. Vergleich im Genus verbi. 
Wie in Abbildung 5 gezeigt wird, kommt die Passivform im Deutschen ungefähr gleich oft wie die Aktivform vor, während im Chinesischen die Aktivform fast doppelt so oft wie die Passivform vorliegt. Das liegt wohl daran, dass bei den deutschen Sätzen in Hinsicht auf Kohäsion entschieden wird, ob Aktiv oder Passiv gewählt wird, und die Wahrscheinlichkeit bei der Topikauswahl ist für die Ernennenden oder für die Ernannten ähnlich. Aber im Chinesischen werden solche Informationen in den meisten Kontexten auf eine unmarkierte Weise geäußert. Nur in bestimmten Kontexten ist die Passivform geeignet, beispielsweise wenn die ernannte Person mit einem längeren Attribut charakterisiert wird, oder wenn am Anfang des Satzes konkret beschrieben wird, wie die fragliche Person gewählt wurde, sollte die ernannte Person als Ausgangspunkt des Satzes gewählt werden.

Durch die Gewohnheit, dass Chinesen in der Muttersprache bei renming sich mehr im Aktiv äußern, sollte die Lehrkraft im Deutschunterricht darauf achten, dass ernennen im Deutschen je nach Kontext auch sehr oft im Passiv gebraucht wird.

Wenn man die Valenzpatterns vergleicht, bekommt man Ergebnisse wie in Abbildung 6:

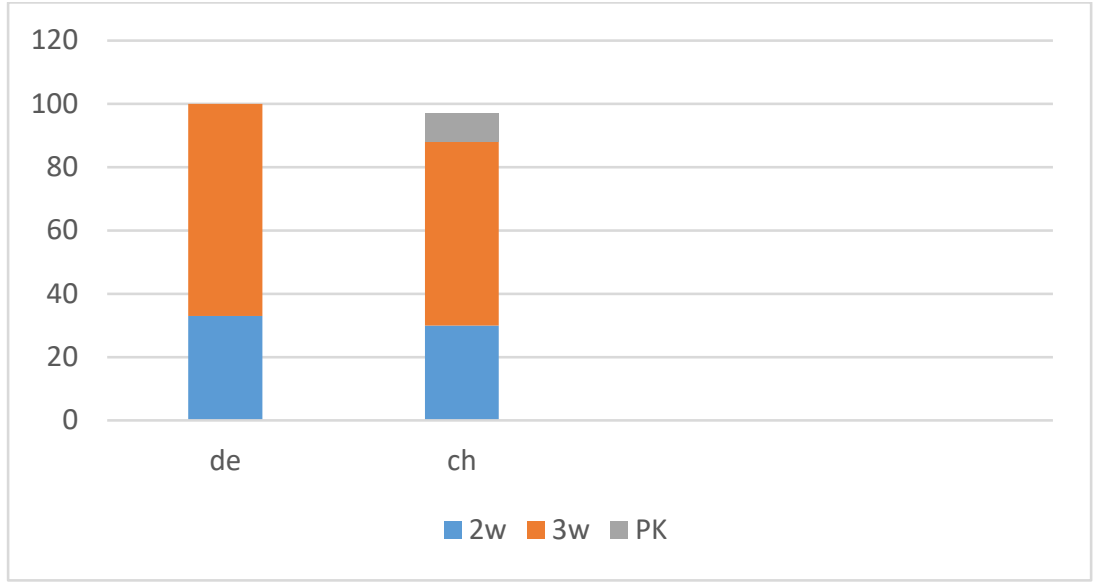

Abbildung 6. Distribution von Valenzpatterns im Vergleich $(2 \mathrm{w}=$ zweiwertig, $3 \mathrm{w}=$ dreiwertig, $\mathrm{PK}=$ Pivotalkonstruktion)

In beiden Sprachen beträgt das zweiwertige Valenzpattern knapp ein Drittel des verbalen Gebrauchs. Unterschiedlich ist nur, dass im Chinesischen ab und zu 
renming in einer Pivotalkonstruktion auftritt (siehe Beispiel 23). Diese Satzstruktur gibt wie das dreiwertige Valenzpattern zuerst Informationen über das Agens (Person/Institution/Organisation, die die Macht übergibt), dann über das Patiens (Person, die die Macht bekommt). Aber im Vergleich zum dreiwertigen Valenzpattern, in der zuletzt die Information über die Stelle betont wird, vermittelt der Sprecher/Schreiber durch Pivotalkonstruktion neben der Stelle die Institution/Organisation oder Gruppen, für die die ernannte Person zuständig ist (siehe Kap. 5.2). Durch diesen Satzbau wird die Macht, die die ernannte Person bekommt, und die Verantwortung, die sie übernimmt, als Folge der Handlung der Ernennung hervorgehoben.

Um zu vermeiden, dass die chinesischen Deutschlernenden diesen Satzbau auf die Zielsprache übertragen und dabei falsche oder ungewöhnliche Sätze bauen, sollte die Lehrkraft betonen, dass ernennen im Deutschen nur zwei Valenzpatterns besitzt. Mögliche Fehler wie ernennen mit dass-Satz oder mit Infinitiv mit $\boldsymbol{z} u$ sollten durch genug Input vermieden werden.

Beim Vergleich des Aktiv-Passiv-Verhältnisses in verschiedenen Valenzpatterns wie in Tabelle 6 sind drei Punkte besonders auffallend:

Tabelle 6. Verhältnisse vom Aktiv und Passiv in verschiedenen Valenzpatterns.

\begin{tabular}{|c|c|c|c|}
\hline & $2 w$ & $3 w$ & PK \\
\hline dt. & $5: 4$ & $4: 5$ & - \\
\hline chin. & $2: 1$ & $3: 2$ & alle aktiv \\
\hline
\end{tabular}

a) Zweiwertigkeit + Aktiv: In dieser Struktur gibt es im Chinesischen Länderoder Ortsnamen als Agens, was im deutschen Korpus nicht festgestellt wurde.

b) Dreiwertigkeit + Passiv: im Deutschen kommen bei der Zweiwertigkeit Aktivsätze etwas häufiger vor als Passivsätze. Aber bei der Dreiwertigkeit nimmt der Anteil von Passivsätzen zu, so dass ihre Anzahl sogar höher ist als die der Aktivsätze. Im Chinesischen fallen die Zahlen etwas anders aus, aber der Anteil der Passivsätze bei Dreiwertigkeit ist höher als bei Zweiwertigkeit. Dieser Trend entspricht dem im Deutschen.

c) Die Pivotalkonstruktion ist nur im Chinesischen vorhanden, und zwar nur im Aktiv. 
Bei der Vermittlung von ernennen kann man deutlich darlegen, welche Lexeme aus welchen Wortfeldern in den Argumentpositionen eingesetzt werden können. Die Lehrkraft sollte darauf achten, die Valenzpatterns mit konkreten semantischen Mustern für Agens und Patiens und für andere Argumente zusammenhängend zu vermitteln. In Bezug auf Punkt b) ist es zudem möglich, durch geeigneten Input die Ausdrücke zu automatisieren. Hinsichtlich Punkt c) ist die Vermittlungsweise eher durch Input und Automatisierung zielführend, was schon oben diskutiert wurde.

Bei Passivsätzen kann man noch beobachten, dass es drei Valenzpatterns gibt, sowohl im Deutschen als auch im Chinesischen. Die möglichen Komponenten sind Patiens, Agens und eine PP, die über die Stelle oder die zu leitende Institution /Gruppe informiert. Vom syntaktischen Gesichtspunkt ist das Verb ernennen /renming entweder zweiwertig oder dreiwertig. Im Aktiv sind Agens und Patiens obligatorisch. Ernennen/renming ohne PP ist zweifelloszweiwertig, mit PP dreiwertig. Aber im Passiv sieht es anders aus. Das obligatorische Patiens nimmt die Rolle des Subjekts an. Von der Frage, ob das Agens im Satz vorhanden ist oder nicht, hängen zwei Varianten der Valenzpatterns ab. Insgesamt kommen vier Varianten vor:
a) Patiens
b) Patiens + Agens
c) Patiens + PP
d) Patiens + Agens + PP

$\mathrm{Zu}$ jeder Variante gibt es Prozentangaben in Tabelle 7.

Tabelle 7. Anteil verschiedener Valenzpatterns von passivischen Sätzen.

\begin{tabular}{|c|l|c|c|}
\hline \multirow{3}{*}{$2 \mathrm{w}$} & Variante & dt. & chin. \\
\cline { 2 - 4 } & a) Patiens & $14 \%$ & $3 \%$ \\
\cline { 2 - 4 } & b) Patiens + Agens & $16 \%$ & $\mathbf{2 \%}$ \\
\cline { 2 - 4 } & Insgesamt & $30 \%$ & $31 \%$ \\
\hline \multirow{3}{*}{$3 \mathrm{w}$} & c) Patiens + PP & $65 \%$ & $53 \%$ \\
\cline { 2 - 4 } & d) Patiens + Agens + PP & $4 \%$ & $\mathbf{1 6 \%}$ \\
\cline { 2 - 4 } & Insgesamt & $69 \%$ & $69 \%$ \\
\hline
\end{tabular}


Tabelle 7 ist zu entnehmen, dass sich die Frequenzen der vier Varianten in beiden Sprachen unterscheiden. Im Passiv ähneln sich die Verhältnisse zwischen Zweiwertigkeit (30\%: $31 \%$ ) und Dreiwertigkeit (69\%: 69\%). Aber wenn wir die Daten näher betrachten, ergibt sich daraus, dass der Anteil der Varianten b und d, die über das Agens deutlich informieren, in den chinesischen Sätzen höher ausfällt. D.h., unter gleichen Umständen tendiert Chinesisch dazu, das Agens, nämlich die Person/Organisation/Institution, die die Macht gibt, zu nennen, im Unterschied zum Deutschen, das mehr Wert auf die Stelle legt.

In unserem Aufsatz ist wird das Tempus als eine wichtige grammatische Kategorie besprochen, aber im Chinesischen wird sehr wenig Wert auf das Tempus gelegt, welches aber eine sehr wichtige Rolle im Deutschen spielt. Deswegen sollte die Lehrkraft sehr darauf achten, dass ernennen im richtigen Tempus verwendet wird. Dabei sind gezielte Inputtexte und Übungen mit dem Tempus notwendig.

\section{Schlussfolgerungen}

Das Ziel dieses Aufsatzes war es, die Valenzpatterns von ernennen und renming aufzustellen und ihre semantischen Interpretationen zu bestimmen. Darüber hinaus wurde auf Implikationen dieses Vorgehens für den Fremdsprachenunterricht und -erwerb hingewiesen. Zuerst wurde das deutsche Verb ernennen und dann das chinesische renming jeweils nach vier grammatischen Kriterien mit Unterstützung von zwei muttersprachlichen Korpora analysiert. Die Analyse, die sich auf Daten aus den zwei Korpora stützt, hat gezeigt, dass sich die zwei Wörter in Bereichen Genus verbi, Valenz, Tempus und Bedeutung unterscheiden. Solche Unterschiede im Gebrauch sind für den Fremdsprachenunterricht und die Lehrwerkentwicklung von Relevanz.

Diese Untersuchung ist jedoch noch hinsichtlich der Tiefe und Systematisierung begrenzt, so dass Möglichkeiten für weitere Explorationen bestehen. Beispielsweise können in weiteren Untersuchungen die Bedeutungen von Argumenten dieser Verben vergleichend analysiert werden. Oder man kann auch andere Verben oder Wörter aus anderen Wortarten anhand der Korpora analysieren, systematisch darstellen und die Zusammenhänge zwischen der Bedeutung der Lexeme und der Grammatik didaktisch aufarbeiten. 
Korpusgestützte Analyse der Verben ernennen und 任命 (renming)

\section{Literatur}

HELBIG, GeRHARD (1982): Valenz - Satzglieder - semantische Kasus - Satzmodelle.

https://www.duden.de/rechtschreibung/ernennen (21.05.2021)

https://www.dwds.de/d/korpora/korpus21 (25.03.2021)

LEMNITZER, LOtHAR / ZinSMEISTER, HeIKe (2015): Korpuslinguistik. Eine Einführung. Tübingen.

LiU, GUOBIN / Du, YAPING (2017): Yuliaoku qudong shijiaoxia de yingyu dongci peijia jiegou yanjiu - yi APPOINT weili [Korpusgestützte Analyse der Valenzpatterns englischer Verben - der Fall von APPOINT]. In: Tianjin waiguoyu daxue xuebao [Journal of Tianjin Foreign Studies University] 24(06):1-7, 78.

Schumacher, Helmut / KubczaK, JaCQueline / Schmidt, Renate / De Ruiter, Vera (2004): VALBU - Valenzwörterbuch deutscher Verben. Tübingen: Narr.

SinClair, John MCH / Renouf, ANTOINETTE (1988): A lexical syllabus for language learning. In: CARTer, Ronald / Channell, JoAnna / MCCARThy, Michael (eds.): Vocabulary and Language Teaching. London, 140-160.

Stopyra, JANUSz (2019): Kollokationen im Dänischen und im Deutschen. Hamburg.

Xue, Fengsheng (1987): Shilun „,ba“ ziju de yuyi texing [Die semantischen Eigenschaften der BA-Sätze]. In: Yuyan jiaохие yи уапjiu [Language Teaching and Linguistic Studies] 1:4-22.

Xun, Endong / Rao, Gaodi / XiaO, Xiaoyue / Zang, Jiaojiao (2016): Dashuju beijingxia BCC yuliaoku de yanzhi [Entwicklung des BCC-Korpus im Kontext von Big Data]. In: Yuliaoku yuyanxue [Corpus Linguistics] 1:93-118.

YAN, LitAO (2018): Hanyu bei ziju de xingcheng jizhi [Der Bildungsmechanismus der BEI-Sätze]. In: Yuwen Yanjiu [Linguistic Research] 149.04:25-32.

Zhen, Fengchao / YAng, Feng (2015): Yuliaoku qudong de xuexizhe yingyu dongci peijia yanjiu [Verb Valency in learner English: A Corpus-driven Study of CONSIDER]. In: Waiguoyu [Journal of Foreign Languages] 6:57-67.

Zhen, Fengchao / Yang, Feng (2016): Peijia jiegou ji dapei peijia zai yingyu cihui jiaoxue Zhong de yingyong: sixiang he fangfa [Application of valency patterns and collovalency: Ideas and Methods]. In: Waiyujie [Foreign Language World] 4:35-42.

(C) by the author, licensee Lodz University - Lodz University Press, Lodz, Poland. This article is an open access article distributed under the terms and conditions of the Creative Commons Attribution license CC BY-NC-ND 4.0 (https://creativecommons.org/licenses/by-nc-nd/4.0/) 